\title{
Ultrafast exciton dynamics reconstruction with a ptychographic approach
}

\author{
Gian Luca Dolso ${ }^{1, *}$, Bruno Moio ${ }^{1,2}$, Giacomo Inzani ${ }^{1}$, Nicola Di Palo ${ }^{1,2}$, Rocío Borrego-Varillas ${ }^{2}$, Mauro Nisoli ${ }^{1,2}$, and \\ Matteo Lucchini ${ }^{1,2}$ \\ ${ }^{1}$ Department of Physics, Politecnico di Milano, 20133 Milano, Italy \\ ${ }^{2}$ Institute for Photonics and Nanotechnologies, IFN-CNR, 20133 Milano, Italy
}

\begin{abstract}
Excitons characterize the ultrafast response of many materials of technological interest. While the development of attosecond science has unlocked the possibility of performing experiments with a suitable timeresolution, the access to the exciton properties remains a non-trivial step. We propose therefore a novel approach to disclose the physical properties behind the ultrafast exciton dynamics based on a phase-retrieval method.
\end{abstract}

\section{Introduction}

Excitons are at the basis of several technological applications in the field of electronics and optoelectronics [1], and their relevance for future devices is expected to grow. The thorough study of their physical properties and optical response is, therefore, a necessary endeavor to achieve their active (optical) control. In this context, attosecond transient absorption and reflection spectroscopies have allowed for the study of ultrafast exciton and electron dynamics in solid-state materials [2-4]. However, the direct extraction of the relevant parameters governing the excitonic response, such as the lifetime and the phonon coupling strength, is a non-trivial procedure and often requires a priori assumptions. Here, we propose a novel method to achieve this goal: ePIX (the extended Ptychographic Iterative engine for eXcitons), based on an iterative ptychographic approach [5].

\section{Methods}

To describe the ultrafast exciton dynamics, we employ the model reported in [6] for atomic-like quasi-particles in solids. The attosecond extreme-ultraviolet (XUV) pulse induces an electronic dipole in the material

$$
O(t) \propto \theta(t) e^{-\frac{t}{T_{e}}} e^{-i \omega_{e} t} e^{\Phi(t)}
$$

where $T_{e}$ is the Auger lifetime of the exciton, $e^{\Phi(t)}$ describes phonon coupling, as discussed in [7], and $\hbar \omega_{e}$ is the transition energy associated to the XUV-induced excitation. $\theta(t)$ is the Heaviside function, that physically describes the instantaneous creation at time $t=0$. The complex dynamic permittivity $\epsilon(\omega, \tau)$ resulting from the interaction of the dipole with an intense infrared (IR) field can be expressed as

$$
\epsilon(\omega, \tau)=\epsilon_{0}+k \int_{-\infty}^{+\infty} O(t) P(t, \tau) e^{-i \omega t} d t
$$

\footnotetext{
*e-mail: gianluca.dolso@polimi.it
}

where $\epsilon_{0}$ is the permittivity response of the crystal structure (for simplicity supposed to be constant), $k$ is a constant and $P(t, \tau)$ is defined as [8]

$$
P(t, \tau)=e^{-\left(\gamma+i \frac{\alpha}{2}\right) \int_{0}^{t} E^{2}\left(t^{\prime}-\tau\right) d t^{\prime}} .
$$

The quantity $P(t, \tau)$ models the interaction of the dipole $O(t)$ with the IR field $E(t)$ through the dynamical Stark shift, and the quantity $\gamma+i \frac{\alpha}{2}$ is the complex polarizability associated to the exciton state [9]. The result of a transient reflection experiment, where the XUV induces the formation of an exciton, can be modelled by applying the Fresnel equations to the permittivity $\epsilon(\omega, \tau)$. The IR vector potential $A(t)$ defines the IR field $E(t)$ and is assumed to be Gaussian within the reconstructing algorithm

$$
A(t)=A_{0} \cos \left\{\omega_{I R} t+\Phi_{I R}+C R t^{2}\right\} e^{-4 \log 2 t^{2} / F W M H^{2}}
$$

where $A_{0}$ is the amplitude, $\omega_{I R}$ is the frequency, $\Phi_{I R}$ is the phase, CR is the chirp rate and FWMH is the amplitude full width at half maximum. Our reconstruction method employs the theoretical model of Eq. (2) to extract $O(t)$ and $E(t)$ from a transient reflection trace. The algorithm is a suitably-modified version of the extended ptychographic iterative engine (ePIE), already employed in the time domain to perform attosecond pulse reconstruction. ePIX, however, significantly differs from ePIE: the constraints set on the object $O(t)$ and probe $P(t)$ must be appropriately recast, in order to apply the algorithm to the new physical context. The reconstruction takes place in two distinct steps within the algorithm, to enhance robustness of the convergence. The first stage (Ptychographic Iterative Engine, PIE) updates the the complex polarizability $\gamma+i \frac{\alpha}{2}$, while keeping the IR field $E(t)$ fixed. The second step (extended Ptychographic Iterative Engine, ePIE) updates the field, within the assumption of a Gaussian temporal profile. If convergence is not reached, it is always possible to iterate this procedure. Figure 1 summarizes the reconstruction process. 


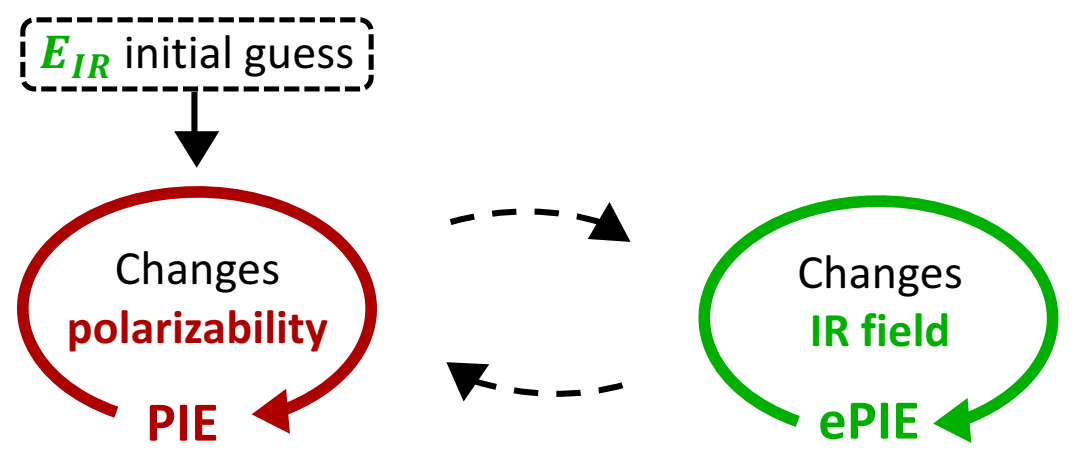

Ptychographic Iterative Engine

extended Ptychographic Iterative Engine

Figure 1. Scheme of the reconstruction algorithm. The reconstruction takes place in two steps: in the first stage (PIE), the algorithm updates the complex polarizability $\gamma+i \frac{\alpha}{2}$; in the second stage (ePIE), the polarizability is kept constant, and the IR field is modified. Both PIE and ePIE update the dipole $O(t)$, associated to the exciton dynamics.
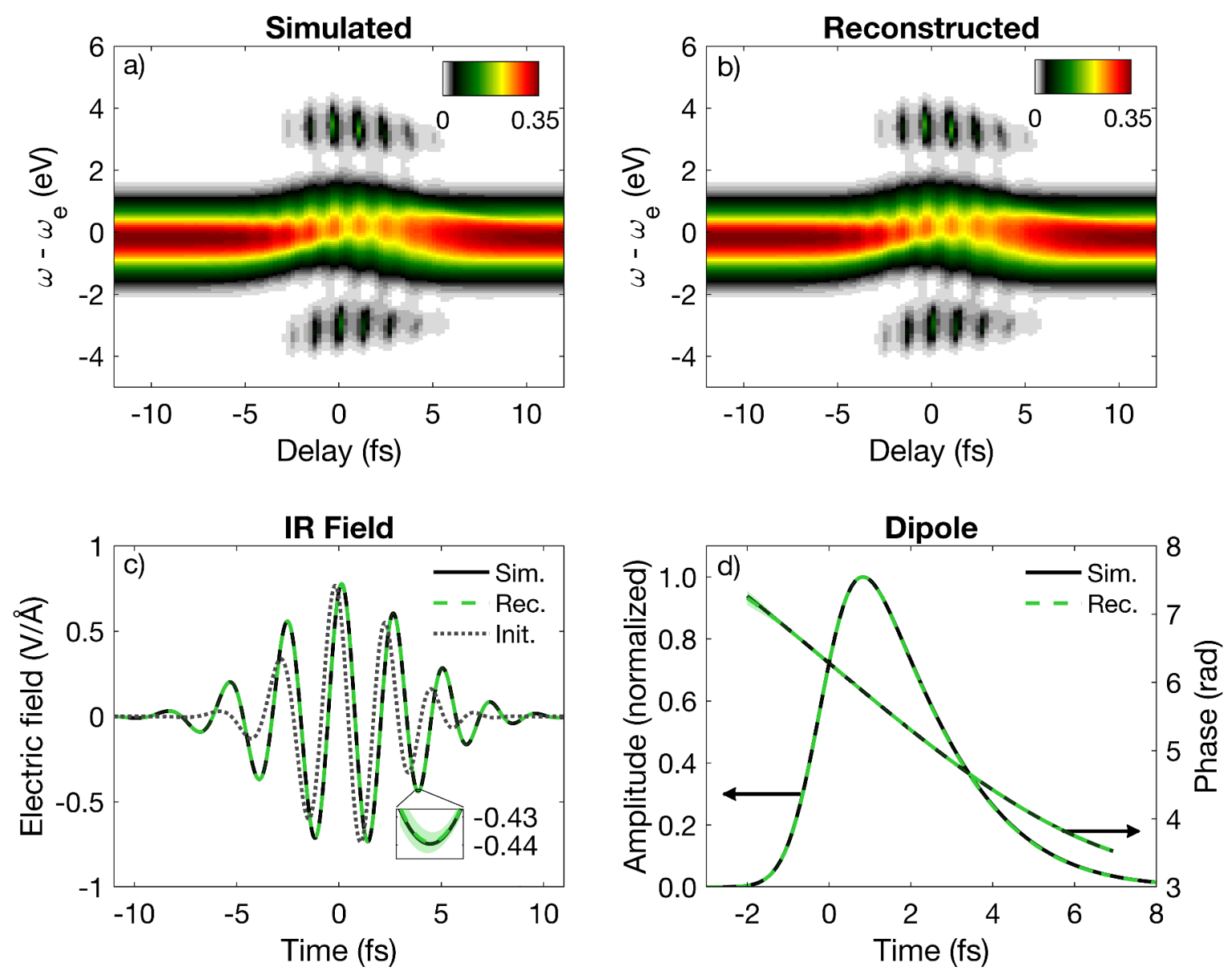

Figure 2. Simulated, a), and reconstructed, b), transient reflectivity trace associated to an excitonic transition, described by the parameters of Table 1. c) Simulated, black solid, and reconstructed, red dashed, dipole moments. d) IR field: simulated, black solid, reconstructed, red dashed, and an example of initial guess, gray dotted. Both amplitude and phase of the dipole moment $O(t)$ are correctly reconstructed. Notice, also, that the ptychographic approach also allows for the retrieval of the IR field. 
Table 1. Simulated and reconstructed parameters, defining the exciton, its interaction with the phonons and with the IR field.

\begin{tabular}{lcc}
\hline Parameter & Sim. & Rec. \\
\hline Amplitude $k\left(\mathrm{fs}^{-1}\right)$ & 30.0 & $30.0 \pm 0.22$ \\
Lifetime $T_{e}(\mathrm{fs})$ & 4.0 & $3.99 \pm 0.076$ \\
Exciton freq. $\omega_{e}\left(\mathrm{rad} \mathrm{fs}^{-1}\right)$ & 84 & $84 \pm 0.0083$ \\
Phonon coupl. $M_{0}\left(\mathrm{fs}^{-1}\right)$ & 0.3 & $0.3 \pm 0.0014$ \\
Phonon freq. $\omega_{0}\left(\mathrm{rad} \mathrm{fs}^{-1}\right)$ & 0.15 & $0.15 \pm 0.0057$ \\
IR FWHM $(\mathrm{fs})$ & 8.0 & $7.99 \pm 0.045$ \\
IR phase $\Phi_{I R}(\mathrm{rad})$ & 1.26 & $1.25 \pm 0.013$ \\
IR chirp CR $\left(\mathrm{fs}^{-2}\right)$ & 0.05 & $0.051 \pm 0.0035$ \\
$\alpha\left(\operatorname{rad~fs} \AA^{-1} \AA^{2}\right)$ & -5.0 & $-5.02 \pm 0.037$ \\
$\gamma\left(\operatorname{rad~fs} \AA^{-1} \AA^{2} \mathrm{~V}^{2}\right)$ & 2.0 & $2.01 \pm 0.022$ \\
\hline
\end{tabular}

\section{Results}

To verify the validity of our approach, we tested ePIX on a set of simulated traces, where we include Auger decay and a finite phonon relaxation. The values used for the simulations are summarized in Table 1. Figure 2a shows the transient reflectance trace, described by the parameters of Table 1 and simulated with Eq. (2). The reconstructed trace obtained with ePIX is shown in Fig. 2b. All features in the transient reflectivity trace are correctly recovered. The comparison between the simulated and reconstructed dipole moments, in Fig. 2c, confirms the accuracy of the reconstruction. Notice that the phase of the dipole is correctly recovered, as well as the modulus. The exciton and phonon parameters can then be determined by fitting the output dipole $O(t)$. The results are reported in Table 1: the indicated errors are standard deviations, obtained by performing 4 reconstructions with different initial guesses. We found the final reconstructed values of all parameters to agree with the simulated with high degree of accuracy. Note that our method also allows for the recovery of the IR field, as shown in Fig. 2d. We have also verified that our method is more accurate when compared to an ordinary non-linear fitting procedure, and requires fewer $a$ priori information on the exciton dynamics, since ePIX makes no assumption regarding the dipole $O(t)$. To further demonstrate that ePIX is a valuable tool for the investigation of exciton dynamics in transient reflectance measurement, we applied the algorithm to different transient reflectance traces, obtained with different choices of the physical parameters: in all cases, the reconstruction accuracy was the same as discussed here.

\section{Conclusions}

We have proved that our novel approach to reconstruct exciton dynamics, ePIX, is effective and precise, and allows to determine the characteristics of the exciton interaction with the phonon population and the external IR field. The method fully exploits the potential and versatility of ptychographic algorithms: the reconstruction of the exciton time-dependent dipole is robust and independent of the initial guess. In addition, the IR field can be characterized within the same reconstruction procedure. ePIX can hence be a useful tool in understanding ultrafast exciton dynamics, paving the way towards a more complete comprehension and control of their optoelectronic properties.

\section{References}

[1] L. V. Butov, Superlattice Microst. 108, 2-26 (2017)

[2] R. Geneaux et al., Philos. T. R. Soc. A 377, 20170463 (2019)

[3] M. Lucchini et al., Nat. Commun. 12, 1021 (2021)

[4] G. D. Lucarelli et al., Rev. Sci. Instrum. 91, 053002 (2020)

[5] M. Lucchini et al., Opt. Express 23, 29502 (2015)

[6] A. Moulet et al., Science 357, 1134-1138 (2017)

[7] G. D. Mahan, Phys. Rev. B 15, 4587 (1977)

[8] A. Wirth et al., Science 334, 195-200 (2011)

[9] R. Géneaux et al., Phys. Rev. Lett. 124, 207401 (2020) 